\title{
Adicción a la cocaína y trastornos de personalidad: un estudio con el MCMI-II
}

\section{Cocaine addiction and personality disorders: a study with the MCMI-II}

IÑAKI LOREA*; JAVIER FERNÁNDEZ-MONTALVO**; José J. LÓPEZ-GOÑI ${ }^{\star *}$; NATALIA LANDA ${ }^{\star \star *}$
${ }^{*}$ Fundación Argibide. Pamplona

**Departamento de Psicología y Pedagogía. Universidad Pública de Navarra

${ }^{* * *}$ Centro de Salud Mental de San Juan. Servicio Navarro de SaludOsasunbidea

Enviar correspondencia a:

Iñaki Lorea. Fundación Argibide. C/ Iturrama 7, entreplanta, 31007 Pamplona-Iruña.

Correo electrónico: i.lorea@fundacionargibide.org

\section{RESUMEN}

En este artículo se lleva a cabo un estudio de los trastornos de personalidad asociados a la adicción a la cocaina. Para ello se contó con una muestra de 60 pacientes cocainómanos, que cumplimentaron el MCMI-II antes del tratamiento, y de 50 sujetos de la población normal con las mismas caracteristicas demográficas (edad, sexo y nivel socioeconómico). El 36,7\% de la muestra clínica (frente al 16\% de la muestra normativa) presentaba, al menos, un trastorno de personalidad. Los trastornos de mayor prevalencia entre los cocainómanos fueron el trastorno pasivo-agresivo de la personalidad y el trastorno dependiente de la personalidad (10\% cada uno de ellos), seguidos del trastorno obsesivo-compulsivo y el histriónico (6,7\% cada uno). Asimismo, el 15\% de la muestra clínica presentaba simultáneamente dos o más trastornos. Por último, se comentan las implicaciones de este estudio para la práctica clínica y para las investigaciones futuras.

Palabras clave: adicción a la cocaína, trastornos de personalidad, comorbilidad, MCMI-II, evaluación.

\section{ABSTRACT}

This paper explores the personality disorders most commonly related to cocaine addiction. The samples used were 60 cocaine-addicted patients, who were assessed with the MCMI-II before treatment, and 50 normative participants from the general population with the same demographic features (age, sex and socioeconomic level). According to the results, $36.7 \%$ of the clinical sample (versus 16\% of the normative sample) showed at least one personality disorder. The most prevalent disorders in the cocaine sample were Passive-Aggressive Personality Disorder and Dependent Personality Disorder (10\% in each case), followed by Obsessive-Compulsive Personality Disorder and Histrionic Personality Disorder (6.7\% each). Moreover, 15\% of the clinical sample showed more than one personality disorder. Finally, the implications of this study for clinical practice and future research in this field are discussed.

Key words: cocaine addiction, personality disorders, comorbidity. MCMI-II, assessment. 


\section{INTRODUCCIÓN}

E estudio de la personalidad y sus trastornos ha sido, y continúa siendo, uno de los terrenos más resbaladizos de la psicopatología. Cualquier clínico suele advertir con frecuencia en sus pacientes comportamientos "anormales", sin que éstos puedan ser diagnosticados como síntomas de cuadros clínicos pertenecientes al Eje I del DSM-IV (Sanjuán y Moltó, 2004).

Los trastornos de personalidad se corresponden con pautas de conducta inflexibles y no adaptativas, estables temporalmente y que reflejan alteraciones globales de la persona (Vázquez, Ring y Avia, 1990). Estos trastornos pueden estar presentes entre el 20 y $40 \%$ de los pacientes psiquiátricos ambulatorios. Hay, por lo tanto, una frecuente comorbilidad entre los trastornos de personalidad y los trastornos mentales (Medina y Moreno, 1998). La presencia de un trastorno de personalidad complica la evolución clínica de un trastorno mental y ensombrece el pronóstico terapéutico (Dowson y Grounds, 1995).

En el ámbito de las adicciones, se ha producido un interés creciente por el estudio de los trastornos duales (Tenorio y Marcos, 2000). El esfuerzo por mejorar las tasas de éxito de los programas de intervención con pacientes adictos ha generado un claro interés por el estudio de las variables que limitan o mediatizan el alcance de dichos programas (Fernández-Montalvo, López-Goñi, Landa, Illescas, Lorea y Zarzuela, 2004; López, Becoña, Lloves, Moneo, Vieitez, Cancelo et al., 2007). En este sentido, ha cobrado una especial importancia -al menos en lo que a las conductas adictivas se refiere- el análisis de las dimensiones de personalidad más frecuentes en este tipo de población (Sáiz, González, Paredes, Martínez y Delgado, 2001) y, más recientemente, de los trastornos concretos de la personalidad que les afectan (Fernández-Montalvo y Echeburúa, 2001; Fernández-Montalvo, Landa, López-Goñi y Lorea, 2006).

La investigación sobre la presencia de los trastornos de personalidad entre los adictos a la cocaína es fundamental para la práctica clínica. Ello se debe a que permite establecer cuatro aspectos esenciales para la intervención terapéutica: a) valorar la probabilidad de implicación en el tratamiento y de cumplimiento de las prescripciones terapéuticas; b) modificar las características del tratamiento para adaptarlas de forma individualizada al paciente; c) establecer apropiadamente los objetivos terapéuticos; y d) determinar la necesidad de una mayor o menor rigidez en la estructuración del contexto terapéutico.

En lo que respecta a esta adicción, sólo recientemente se ha comenzado a valorar la prevalencia de trastornos de personalidad entre los pacientes afectados (FernándezMontalvo y Lorea, 2007; López y Becoña, 2006). Los resultados obtenidos hasta la fecha indican una gran presencia de trastornos de personalidad entre los adictos a la cocaína, que afectan, como media, en torno al 66\% de los casos (2 de cada 3 pacientes) según los estudios llevados a cabo (Barber, Frank, Weiss, Blaine, Siqueland, Moras et al., 1996; Fernández-Montalvo, Lorea, López-Goñi y Landa, 2003; Kranzler, Satel y Apter, 1994; López, Becoña, Casete, Lage, Díaz, García, et al., (2007); Pedrero, Puerta, Lagares y Sáez, 2003; Sonne y Brady, 1998) (tabla 1). Sin embargo, se observa una amplia heterogeneidad de trastornos, que no permite establecer, hoy por hoy, un perfil homogéneo de personalidad en este tipo de pacientes.

Tabla 1. Trastornos de personalidad en la adicción a la cocaína

\begin{tabular}{|c|c|c|c|}
\hline AUTORES & N & CUESTIONARIO & PREVALENCIA \\
\hline Calsyn y Saxon (1990) & 37 & MCMI & $97 \%$ \\
\hline Craig (2000) & 283 & MCMI-III & $91 \%$ \\
\hline $\begin{array}{c}\text { Fernández-Montalvo et al. } \\
\text { (2003) }\end{array}$ & 35 & MCMI-II & $45,7 \%$ \\
\hline $\begin{array}{c}\text { Pedrero et al. (2003) } \\
\text { López et al. (2007) }\end{array}$ & 32 & MCMI-II & $65,6 \%$ \\
\hline AUTORES & N & MCMI-II & $66,6 \%$ \\
\hline Weiss et al. (1993) & 50 & SCID-II & $74 \%$ \\
\hline Kranzler et al. (1994) & 50 & SCID-II & $70 \%$ \\
\hline Barber et al. (1996) & 289 & SCID-II & $47,1 \%$ \\
\hline Sonne y Brady (1998) & 47 & SCID-II & $66,7 \%$ \\
\hline Sanz y Larrazábal (2002) & 65 & IPDE & $64,3 \%$ \\
\hline
\end{tabular}

El objetivo de este estudio es determinar la comorbilidad de los trastornos de personalidad en un grupo de pacientes adictos a la cocaína que acude a consulta en busca de tratamiento. Este trabajo forma parte de un estudio más amplio sobre las características de consumo, psicopatológicas y neurocognitivas de los pacientes cocainómanos. Se trata, en último término, de identificar los trastornos concretos que afectan en mayor medida a este tipo de pacientes para, en una fase posterior, adaptar los programas terapéuticos a las necesidades específicas de los pacientes cocainómanos.

\section{MÉTODO}

\section{Sujetos}

La muestra de este estudio está compuesta por 110 sujetos (60 pacientes adictos a la cocaína y 50 personas de la población normal). Por lo que se refiere al grupo clínico, se trata de 60 pacientes que acudieron en busca de ayuda terapéutica al programa de tratamiento ambulatorio para la adicción a sustancias estimulantes de Proyecto Hombre de Navarra, durante el período comprendido entre mayo de 2004 y mayo de 2007. De acuerdo con los criterios de admisión al estudio, los pacientes tenían que: a) cumplir los criterios diagnósticos del DSM-IV-TR (American Psychiatric Association, 2000) para la dependencia de la cocaína; b) tener una edad comprendida entre los 18 y 65 años; 
c) no ser diagnosticado de dependencia actual a otras sustancias (con la excepción de la nicotina), ni de trastornos del espectro esquizofrénico, según criterios diagnósticos del DSM-IV-TR (APA, 2000); y d) dar su consentimiento informado para participar en el estudio.

Por su parte, el grupo de control $(\mathrm{N}=50)$ se seleccionó con la finalidad de servir de comparación con el grupo clínico en las pruebas de personalidad (MCMI-II). Se trataba de personas sin problemas de consumo de drogas (evaluado mediante una entrevista personal), apareadas en edad, sexo y nivel cultural con el grupo experimental. Esta muestra se seleccionó entre los pacientes ingresados en la Clínica Ubarmin por diferentes problemas traumatológicos. Un resumen de las características sociodemográficas de ambas muestras puede observarse en la Tabla 2.

Tabla 2. Características sociodemográficas de la muestra

\begin{tabular}{|c|c|c|}
\hline \multirow[t]{2}{*}{ Variables } & $\begin{array}{c}\text { Adictos a la cocaína } \\
\qquad(\mathrm{N}=60)\end{array}$ & $\begin{array}{l}\text { Grupo de control } \\
\qquad(\mathrm{N}=50)\end{array}$ \\
\hline & $\mathrm{N} \quad(\%)$ & $\mathrm{N} \quad(\%)$ \\
\hline Edad Media (rango) & $31,03(20-46)$ & $32,48(22-44)$ \\
\hline \multicolumn{3}{|l|}{ Sexo: } \\
\hline Hombre & $50(83,3 \%)$ & $38(76 \%)$ \\
\hline Mujer & $10(16,7 \%)$ & $12(24 \%)$ \\
\hline Estado civil: & \multirow{4}{*}{$\begin{array}{c}41(68,3 \%) \\
11(18,3 \%) \\
8(13,3 \%)\end{array}$} & \multirow{4}{*}{$\begin{array}{c}13(26 \%) \\
32(64 \%) \\
5(10 \%)\end{array}$} \\
\hline Soltero & & \\
\hline Casado/conviviendo & & \\
\hline Separado/divorciado & & \\
\hline \multicolumn{3}{|l|}{ Nivel cultural: } \\
\hline Sin estudios & $7 \quad(11,7 \%)$ & 7 (14\%) \\
\hline Estudios primarios & $22(36,7 \%)$ & $15(30 \%)$ \\
\hline Estudios secundarios & $26(43,3 \%)$ & $20(40 \%)$ \\
\hline Estudios universitarios & $5 \quad(8,3 \%)$ & $8(16 \%)$ \\
\hline Situación laboral: & \multirow[b]{2}{*}{$44(73,3 \%)$} & \multirow[b]{2}{*}{$41(82 \%)$} \\
\hline En activo & & \\
\hline Desempleado & $11(18,3 \%)$ & $4 \quad(8 \%)$ \\
\hline ILT & $5 \quad(8,3 \%)$ & -- \\
\hline Pensionista & -- & $3(6 \%)$ \\
\hline Ama de casa & -- & $2(4 \%)$ \\
\hline
\end{tabular}

Por lo que se refiere a las principales características sociodemográficas de la muestra clínica, la edad media era de 31,1 años (con un rango que oscilaba entre los 20 y los 46 años), 50 sujetos $(83,3 \%)$ eran hombres y $10(16,7 \%)$ mujeres. Desde una perspectiva laboral, la mayor parte de los sujetos de la muestra (el 81,6\%) se encontraba en activo.

Por lo que se refiere al consumo de cocaína, la edad media del primer consumo se situaba en los 20,4 años, y el consumo medio actual era de 6,7 gramos semanales. Una descripción más detallada de las características de consumo de esta muestra, así como de la gravedad de la adicción y de la sintomatología psicopatológica asociada puede encontrarse en el estudio de Fernández-Montalvo, Lorea, López-Goñi y Landa (2008).

\section{Instrumentos de evaluación}

Todos los pacientes de la muestra fueron evaluados mediante una Entrevista Clínica en la que se recogían, por una parte, las variables sociodemográficas y, por otra, los datos relativos al consumo de cocaína. Asimismo, en esta entrevista se incluían los criterios diagnósticos del DSM-IVTR (APA, 2000) para la dependencia de cocaína.

Por otra parte, la evaluación de los trastornos de personalidad se llevó a cabo el Inventario Clínico Multiaxial de Millon (MCMI-II) (Millon, 1997). Se trata de un cuestionario clínico autoaplicado de evaluación de la personalidad y de distintos sindromes clínicos. Está compuesto por 175 ítems de respuesta dicotómica (verdadero/falso), que proporcionan información sobre 10 escalas básicas de personalidad (esquizoide, fóbica, dependiente, histriónica, narcisista, antisocial, agresivosádica, compulsiva, pasivo-agresiva y autodestructiva), 3 escalas de personalidad patológica (esquizoide, límite y paranoide), 6 sindromes clínicos de gravedad moderada (ansiedad, histeriforme, hipomanía, distimia, abuso de alcohol y abuso de drogas) y 3 síndromes clínicos de gravedad severa (pensamiento psicótico, depresión mayor y trastorno delirante). Debido al objetivo de este estudio, se presentan solamente los resultados de las 13 escalas de personalidad.

\section{Procedimiento}

La evaluación de todos los sujetos de la muestra clínica se llevó a cabo en el marco de la evaluación pretratamiento. De esta forma, a medida que los pacientes acudian al programa, se llevaba a cabo la entrevista clínica y se cumplimentaba el MCMI-II.

En concreto, se llevaron a cabo 3 sesiones de evaluación. En la primera se recogian los datos sociodemográficos, en la segunda se valoraba la severidad de la adicción y, por último, en la tercera sesión se evaluaban las características de personalidad de los pacientes de la muestra. En este sentido, tal y como se ha sugerido por parte de algunos autores (Sonne y Brady, 1998), la evaluación de las características de personalidad se realizó una vez transcurridas 3 semanas desde el inicio de la abstinencia, para eliminar así la posible influencia de síntomas de deprivación.

La evaluación de los sujetos normativos se llevó a cabo, tras una estratificación previa en edad, sexo y clase social, en una única sesión, en la que sólo se valoraban los trastornos de personalidad con el MCMI-II.

Con arreglo a los criterios más conservadores de Weltzer (1990), en este estudio sólo se ha considerado la presencia de un trastorno de personalidad cuando la puntuación en la tasa-base (TB) del MCMI-/l era superior a 84 . 


\section{Análisis estadísticos}

Todos los análisis estadísticos de este estudio han sido llevados cabo con el SPSS (versión 15.0 para Windows). Para determinar las características de la muestra se han utilizado análisis estadísticos de carácter descriptivo (porcentajes, medias y desviaciones típicas). Asimismo, la comparación entre los grupos se ha llevado a cabo mediante la prueba de Chi cuadrado, en el caso de las variables categóricas, y la $U$ de Mann-Whitney, como prueba no paramétrica, en el caso de las variables cuantitativas. Las diferencias encontradas se han considerado significativas a partir de una $p<0,05$.

\section{RESULTADOS}

Los resultados obtenidos con el MCMI-II muestran que 22 sujetos (el 36,7\% de la muestra clínica) obtuvieron puntuaciones indicadoras de la existencia de, al menos, un trastorno de la personalidad. Los observados con mayor frecuencia fueron el trastorno pasivo-agresivo de la personalidad y el trastorno dependiente la personalidad (con una tasa del 10\% cada uno de ellos). Les siguen, por orden de frecuencia, el trastorno obsesivo-compulsivo y el histriónico, con 4 casos cada uno (un 6,7\% del total), y los trastornos esquizoide, fóbico, narcisista y agresivo-sádico, con 3 casos (el 5\% del total de la muestra) cada uno. Los trastornos menos representados fueron el antisocial y límite, con 2 casos (3,3\%), y el trastorno autodestructivo de la personalidad, que afectó sólo a 1 caso (el 1,7\%). Los trastornos esquizotípico y paranoide no aparecieron representados en esta muestra.

Asimismo, un 15\% de la muestra clínica presentaba simultáneamente dos o más trastornos y un $6 \%$ más de tres. Un resumen de los resultados de prevalencia de cada trastorno de personalidad, así como la comparación con la población normal, se expone en la tabla 3.

En el caso de la población normal, la tasa de prevalencia de los trastornos de la personalidad asciende al 16\% (8 sujetos). Entre ellos, destacan ligeramente el trastorno fóbico y el trastorno por dependencia, con una tasa del $4 \%$ cada uno de ellos.

La comparación de la tasa global de trastornos entre ambas submuestras arroja la existencia de diferencias estadísticamente significativas $\left(\chi^{2}=4,87 ; p<0,05\right)$. Más en concreto, los cocainómanos presentan una tasa de prevalencia de trastornos de personalidad significativamente superior a la muestra normativa.

Por otra parte, si se comparan los datos obtenidos en función del sexo, los resultados muestran la ausencia de diferencias significativas. En concreto, la mitad de las mujeres de la muestra (50\%) presenta un trastorno de personalidad, frente al 34\% de los hombres. Sin embargo, estas diferencias, importantes desde un punto de vista clínico, no lo son desde una perspectiva estadística $\left(\chi^{2}=0,92\right.$; $p<0,54)$. En cualquier caso, es necesario tener en cuenta el
Tabla 3. Comparación entre el grupo clínico y el grupo de control en el MCMI-II

\begin{tabular}{|c|c|c|c|c|}
\hline \multirow{2}{*}{$\begin{array}{l}\text { TRASTORNOS DE } \\
\text { PERSONALIDAD }\end{array}$} & $\begin{array}{c}\text { ADICTOS A LA } \\
\text { COCAÍNA } \\
(\mathrm{N}=60)\end{array}$ & \multicolumn{2}{|c|}{$\begin{array}{c}\text { GRUPO DE } \\
\text { CONTROL } \\
(\mathrm{N}=50)\end{array}$} & \multirow[t]{2}{*}{$\chi^{2}$} \\
\hline & $\mathrm{N} \quad(\%)$ & & (\%) & \\
\hline Esquizoide & $3 \quad(5 \%)$ & & -- & -- \\
\hline Fóbica & $3 \quad(5 \%)$ & 2 & $(4 \%)$ & 0,04 \\
\hline Dependiente & $6(10 \%)$ & 2 & $(4 \%)$ & 0,7 \\
\hline Histriónica & $4(6,7 \%)$ & 1 & $(2 \%)$ & 0,5 \\
\hline Narcisista & $3 \quad(5 \%)$ & & -- & -- \\
\hline Antisocial & $2(3,3 \%)$ & & -- & -- \\
\hline Agresiva & $3 \quad(5 \%)$ & & -- & -- \\
\hline Compulsivo & $4(6,7 \%)$ & 1 & $(2 \%)$ & 0,5 \\
\hline Pasiva & $6(10 \%)$ & 1 & $(2 \%)$ & 1,7 \\
\hline Autodestructiva & $1(1,7 \%)$ & & -- & -- \\
\hline Esquizotípica & -- & & -- & -- \\
\hline Límite & $2(3,3 \%)$ & 1 & $(2 \%)$ & 0,02 \\
\hline Paranoide & -- & & -- & -- \\
\hline TOTAL ${ }^{1}$ & $22 \quad(36,7 \%)$ & 8 & $(16 \%)$ & $4,87^{*}$ \\
\hline
\end{tabular}

\section{${ }^{*} p<0,05$}

'NOTA: El número total de sujetos afectados por trastornos de personalidad es inferior a la suma total de trastornos debido a que hay pacientes que presentan más de un trastorno de personalidad asociado.

Tabla 4. Comparación de puntuaciones en el MCMI-Il según sexo

\begin{tabular}{|c|c|c|c|c|}
\hline Escala & $\begin{array}{c}\begin{array}{c}\text { HOMBRE } \\
(\mathrm{N}=50)\end{array} \\
\text { Media (DT) }\end{array}$ & $\begin{array}{c}\text { MUJER } \\
(\mathrm{N}=10) \\
\text { Media (DT) }\end{array}$ & $\begin{array}{c}\text { U de } \\
\text { Mann- } \\
\text { Whitney }\end{array}$ & Z \\
\hline Esquizoide & $54,28(21,42)$ & $45,2(17,27)$ & 179,5 & $-1,40$ \\
\hline Fóbica & $45,96(24,38)$ & $60(10,77)$ & 152 & $-1,94$ \\
\hline Dependiente & $59,3(21,81)$ & $59,4(24,86)$ & 244 & $-0,12$ \\
\hline Histriónica & $55,02(19,56)$ & $75(12,06)$ & 95 & $-3,08^{* *}$ \\
\hline Narcisista & $48,94(24,04)$ & $66,4(18,06)$ & 136 & $-2,26^{*}$ \\
\hline Antisocial & $50,84(22,33)$ & $69,3(12,26)$ & 139,5 & $-2,19^{*}$ \\
\hline Agresiva & $50,66(22,66)$ & $70(6,73)$ & 93 & $-3,12^{* *}$ \\
\hline Compulsiva & $53,22(20,84)$ & $48,8(19,49)$ & 225 & $-0,50$ \\
\hline Pasivo-agresiva & $44,28(28,57)$ & $66,5(11,86)$ & 100,5 & $-2,97^{* *}$ \\
\hline Autodestructiva & $44,46(20,35)$ & $60,7(14,29)$ & 119 & $-2,60^{* *}$ \\
\hline Esquizotípica & $40,4(22,10)$ & $46,4(10,63)$ & 223,5 & $-0,53$ \\
\hline Límite & $42,82(26,32)$ & $60,5(5,62)$ & 140,5 & $-2,17^{*}$ \\
\hline Paranoide & $53,84(17,52)$ & $66(6,16)$ & 120 & $-2,58^{* *}$ \\
\hline
\end{tabular}

${ }^{*} p<0,05 ;{ }^{* *} p<0.01$ 
reducido tamaño de la muestra de mujeres para interpretar este resultado.

No obstante, cuando se compara desde una perspectiva cuantitativa las puntuaciones medias del MCMI-II entre los hombres y las mujeres (tabla 4), los resultados arrojan diferencias significativas en las escalas histriónica, narcisista, antisocial, agresivo-sádica, pasivo-agresiva, autodestructiva, límite y paranoide. En todos los casos, estas diferencias apuntan a mayores puntuaciones en el grupo de mujeres que en el grupo de hombres.

\section{DISCUSIÓN}

En este estudio se ha llevado a cabo, en primer lugar, un análisis de los trastornos de personalidad que presenta una muestra clínica de adictos a la cocaína, y en segundo lugar, se ha comparado la tasa encontrada con un grupo de control procedente de la población normal. Los resultados obtenidos muestran la existencia de una mayor proporción de trastornos de la personalidad en el grupo de adictos a la cocaína que en el grupo de control. Este dato corrobora los datos ya encontrados en otros estudios que muestran una alta presencia de trastornos de personalidad en los pacientes cocainómanos (cfr. Fernández-Montalvo y Lorea, 2007; López y Becoña, 2006).

Más en concreto, la tasa de comorbilidad obtenida en el grupo de adictos es del 36,7\%. Esta tasa, aunque importante -aproximadamente, uno de cada tres cocainómanos que acuden a consulta presenta como mínimo un trastorno de la personalidad-, se sitúa por debajo de las obtenidas en otros estudios publicados, que arrojan tasas de prevalencia que oscilan entre el 45\% (FernándezMontalvo et al., 2003) y el $97 \%$ (Calsyn y Saxon, 1990). Probablemente, ello se relacione con los criterios más restrictivos que se han establecido en este estudio para determinar la presencia de un trastorno de personalidad: principalmente la obtención de un Tasa Base superior a 84. Las mayores tasas encontradas en otros estudios realizados en nuestro entorno con el mismo instrumento pueden estar relacionadas con la elección de un punto de corte inferior para determinar la presencia de un trastorno de la personalidad. Además es posible que, al tratarse de un tratamiento ambulatorio, los sujetos afectados por alteraciones de la personalidad más graves sean atendidos en tratamientos que ofrecen una mayor contención, como por ejemplo una comunidad terapéutica.

Por lo que se refiere a los trastornos de personalidad concretos con mayor prevalencia, destaca la ausencia de los trastornos más "clásicos" encontrados habitualmente en las conductas adictivas: principalmente los del tipo B (el límite y el antisocial). La disparidad e imprecisión de los criterios diagnósticos para los trastornos de personalidad podría explicar, en parte, la divergencia de resultados obtenidos. Asimismo, no se ha encontrado un trastorno de la personalidad específico que se relacione con la adicción a la cocaina, sino que parece que las alteraciones de la personalidad en general podrían constituir un factor de riesgo en el desarrollo de cualquier trastorno adictivo. Además, las conductas más características de la adicción a la cocaina (y a cualquier sustancia) podrían hacer más disfuncionales algunos rasgos de la personalidad.

Los trastornos de personalidad más prevalentes obtenidos en este estudio son el trastorno de la personalidad por dependencia y el trastorno pasivo-agresivo de la personalidad. En el primer caso, se trata de sujetos con una sensación básica de indefensión e inseguridad, que tratan de compensar a través de la búsqueda de afecto, seguridad y consejo en sus relaciones (Millon, 1996). En estos casos, el consumo de cocaína podría provocar un impacto diferencial, al mejorar de manera determinante, aunque transitoria, la sensación básica de competencia vital (Newlin, 2002).

Por lo que se refiere al trastorno pasivo-agresivo de la personalidad, se trata de personas caracterizadas por sentimientos frecuentes de duda y de vacilación entre seguir los propios deseos o los de los demás. Su conducta suele mostrarse errática y afectada por fuertes impulsos discrepantes entre sí. Esto coincide con la ambivalencia motivacional que se observa entre los adictos a la cocaína que acuden a consulta. En el ámbito clínico, es frecuente que el paciente reconozca los efectos negativos de la cocaina, aunque, por otra parte, mantenga la expectativa de llegar a controlar el consumo, sin tener que abandonar totalmente el uso de la sustancia (Gawin, Khalsa y Ellinwood, 1997). En otras palabras, muchos pacientes quieren dejar de tener problemas por el consumo de cocaina, y no tanto dejar de consumirla. Esta hipótesis podría suponer un posible punto de encuentro entre el trastorno pasivo-agresivo y la adicción a la cocaína. En cualquier caso, se trata de un trastorno que ya ha mostrado su relación en estudios previos con la dependencia de cocaína en particular (Fernández-Montalvo et al., 2003; López et al., 2007; Pedrero et al., 2003). Ello ha llevado a desarrollar distintas hipótesis explicativas de la relación entre el abuso de drogas y la presencia del trastorno pasivo-agresivo (cfr. Pedrero, López y Olivar, 2006), sin que hasta la fecha haya datos concluyentes.

En cualquier caso, los resultados de esta investigación reflejan un índice de comorbilidad muy elevado entre la dependencia de la cocaína y los trastornos de personalidad. Al margen de las limitaciones terapéuticas existentes en el tratamiento de los trastornos de personalidad (Echeburúa y Corral, 1999; Echeburúa y Fernández-Montalvo, 2003), resulta imprescindible tenerlos en consideración cuando se tiene que planificar una intervención en pacientes adictos (Cangas y Olivencia, 1999; Cervera, Bolinches y Valderrama, 1999).

No obstante, este estudio presenta algunas limitaciones. En primer lugar, el tamaño de la muestra de cocainómanos, aunque relevante desde un punto de vista clínico, es relativamente pequeño desde una perspectiva estadística. Por otra parte, la valoración de los trastornos de personalidad se ha llevado a cabo con una prueba de autoinforme: el MCMI-II. A pesar de la utilidad de este 
instrumento, y de su amplia utilización en el ámbito de las conductas adictivas, la utilización de autoinformes tiende a producir un sobrediagnóstico de trastornos de personalidad (cfr. Fernández-Montalvo y Echeburúa, 2006). Además, en el caso del MCMI no todos los estudios utilizan la misma tasa base como punto de corte. Parece necesario recurrir, en investigaciones futuras, a entrevistas clínicas especificas de los trastornos de personalidad -el IPDE (Loranger, 1995) o la SCID-II (First, Spitzer, Gibbon, Williams y Benjamin, 1994), por ejemplo-. Ello permitirá, tal y como ya se ha puesto de manifiesto en otras adicciones (cfr. Fernández-Montalvo y Echeburúa, 2004; Landa, Fernández-Montalvo, López-Goñi y Lorea, 2005) el diagnóstico más preciso de este grupo de trastornos y, como consecuencia, la reducción de la amplia variabilidad observada en los resultados de los diferentes estudios sobre los trastornos de personalidad en los adictos a la cocaína.

\section{AGRADECIMIENTOS}

Este estudio se ha financiado con un proyecto de investigación del Departamento de Salud del Gobierno de Navarra (código 2/2006)

\section{REFERENCIAS}

American Psychiatric Association, (2000). Diagnostic and statistical manual of mental disorders ( $4^{\text {th }}$ ed. rev). Washington, D. C. APA.

Barber, J. B., Frank, A., Weiss, R. D., Blaine, J., Siqueland, L., Moras, K., et al. (1996). Prevalence and correlates of personality disorder diagnoses among cocaine dependent outpatients. Journal of Personality Disorders, 10, 297-311.

Calsyn, D. A. y Saxon, A. J. (1990). Personality disorder subtypes among cocaine and opioid addicts using the Millon Clinical Multiaxial Inventory. International Journal of the Addictions, 25, 10371049.

Cangas, A. y Olivencia, J. J. (1999). Alteraciones de la personalidad asociadas a las conductas adictivas. Apuntes de Psicología, 17, 109-116.

Cervera, G., Bolinches, F. y Valderrama, J. C. (1999). Trastornos de la personalidad y drogodependencias. Trastornos Adictivos, 1, 91-99.

Craig, R. J. (2000). Prevalence of personality disorders among cocaine and heroin addicts. Substance Abuse, 21, 87-94.

Dowson, J. H. y Grounds, A. T. (1995). Personality disorders. Recognition and clinical management. Cambridge. Cambridge University Press.

Echeburúa, E. y Corral, P. (1999). Avances en el tratamiento cognitivoconductual de los trastornos de personalidad. Análisis y Modificación de Conducta, 25, 585-614.

Echeburúa, E. y Fernández-Montalvo, J. (2003). Terapia de conducta dialéctica y trastorno limite de la personalidad: aplicaciones clínicas. En V. Rubio y A. Pérez (Eds.), Trastornos de la personalidad (pp. 319-327). Madrid: Elsevier.
Fernández-Montalvo, J. y Echeburúa, E. (2001). Trastornos de personalidad y juego patológico: una revisión crítica. Psicología Conductual, 9, 527-539.

Fernández-Montalvo, J. y Echeburúa, E. (2004). Pathological gambling and personality disorders: An exploratory study with the IPDE. Journal of Personality Disorders, 18, 500-505.

Fernández-Montalvo, J. y Echeburúa, E. (2006). Uso y abuso de los autoinformes en la evaluación de los trastornos de personalidad. Revista de Psicopatología y Psicología Clínica, 11, 1-12.

Fernández-Montalvo, J., Landa, N., López-Goñi, J. J. y Lorea, I. (2006). Personality disorders in alcoholics: A comparative pilot study between the IPDE and the MCMI-II. Addictive Behaviors, 31, 1442-1448.

Fernández-Montalvo J., López-Goñi, J. J., Landa, N., Illescas, C., Lorea I. y Zarzuela, A. (2004). Trastornos de personalidad y abandonos terapéuticos en pacientes adictos: resultados en una comunidad terapéutica. International Journal of Clinical and Health Psychology, 2, 271-283.

Fernández-Montalvo, J. y Lorea, I. (2007). Comorbilidad de la adicción a la cocaína con los trastornos de la personalidad. Anales del Sistema Sanitario de Navarra, 30, 225-231.

Fernández-Montalvo, J., Lorea, I., López-Goñi, J. J. y Landa, N. (2003). Trastornos de personalidad en adictos a cocaína: un estudio piloto. Análisis y Modificación de Conducta, 29, 79-98.

Fernández-Montalvo, J., Lorea, I., López-Goñi, J. J. y Landa, N. (2008). Comorbilidad psicopatológica en la adicción a la cocaina: Resultados con el SCL-90-R. Behavioral Psychology/Psicología Conductual, 16, 273-286.

First, M. B., Spitzer, R. L., Gibbon, M., Williams, J. B. W. y Benjamin, L. (1994). Structured Clinical Interview for DSM-IV Axis II Personality Disorders (SCID-II). New York: New York State Psychiatric Institute, Biometrics Research Department.

Gawin, F. H., Khalsa, M. E. y Ellinwood, E. (1997). Estimulantes. En M.C. Galanter y H. D. Kleber, (Eds.), Tratamiento de los trastornos por abuso de sustancias. Barcelona. Masson.

Kranzler, H. R., Satel, S. y Apter, A. (1994). Personality disorders and associated features in cocaine-dependent inpatients. Comprehensive Psychiatry, 35, 335-340.

Landa, N., Fernández-Montalvo, J., López-Goñi, J. J. y Lorea, I. (2005). Trastornos de personalidad en alcohólicos: un estudio con el IPDE. Psicología Conductual, 13, 275-288.

López, A. y Becoña, E. (2006). Patrones y trastornos de personalidad en personas con dependencia de la cocaína en tratamiento. Psicothema, 18, 578-583.

López, A., Becoña, E., Lloves, M., Moneo, A., Vieitez, I., Cancelo, J., et al., (2007). Evolución del tratamiento y consumo en personas con dependencia de la cocaina, a los doce meses, en función de la psicopatología asociada y el funcionamiento cotidiano. Adicciones, 19, 251-266.

López, A., Becoña, E., Casete, L., Lage, M. T., Díaz, E., García, J. M. et al., (2007). Dependencia de la cocaina y trastornos de personalidad. Análisis de su relación en una muestra clínica. Trastornos Adictivos, 9, 215-227.

Loranger, A. W. (1995). International Personality Disorder Examination (IPDE). Ginebra. Organización Mundial de la Salud.

Medina, A. y Moreno, M. J. (1998). Los trastornos de la personalidad. Un estudio médico-filosófico. Córdoba. Nanuk.

Millon, T. (1996). Disorders of Personality. New York. Wiley and Sons. 
Millon, T. (1997). Millon Clinical Multiaxial Inventory-II (MCMI-II). Minneapolis. National Computer Systems.

Newlin, D. B. (2002). The self-perceived survival ability and reproductive fitness (SPFit) theory of substance use disorders. Addiction, 97, 427-445.

Pedrero, E. J., López, A. y Olivar, A. (2006). El trastorno negativista de la personalidad y su relación con el abuso de sustancias. Trastornos Adictivos, 8, 22-41.

Pedrero, E. J., Puerta, C., Lagares, A. y Sáez, A. (2003).Prevalencia e intensidad de trastornos de personalidad en adictos a sustancias en tratamiento en un centro de atención a las drogodependencias. Trastornos Adictivos, 5, 241-255.

Sáiz, P. A., González, M. P., Paredes, B., Martínez, S. y Delgado, J. M. (2001). Personalidad y uso-abuso de cocaina. Adicciones, 13 (suplemento 2), 47-59.

Sanjuán, J. y Moltó, M. D. (2004). Trastornos de la personalidad: genética y evolución. En M. Roca (coord.), Trastornos de personalidad. Barcelona. Psiquiatría Editores.
Sanz, J. y Larrazabal, A. (2002). Comorbilidad de dependencia de cocaína y trastornos de personalidad. Implicaciones clínicas y pronósticas. Interpsiquis.

Sonne, S. C. y Brady, K. T. (1998). Diagnosis of personality disorders in cocaine-dependent individuals. The American Journal on Addictions, 7, 1-6.

Tenorio, J. y Marcos, J. A. (2000). Trastornos duales: tratamiento y coordinación. Papeles del Psicólogo, 77, 58-63.

Vázquez, C., Ring, J. y Avia, M. D. (1990). Trastornos de la personalidad. En F. Fuentenebro y C. Vázquez (Eds.). Psicología Médica, Psicopatología y Psiquiatría. Madrid. Interamericana-McGraw-Hill, vol. 2².

Weiss, R. D., Mirin, S. M., Griffin, M. L., Gunderson, J. G. y Hufford, C. (1993). Personality disorders in cocaine dependence. Comprehensive Psychiatry, 34, 145-149.

Weltzer, S. (1990). The Millon Clinical Multiaxial Inventory (MCMI): a review. Journal of Personality Assessment, 55, 445-464. 


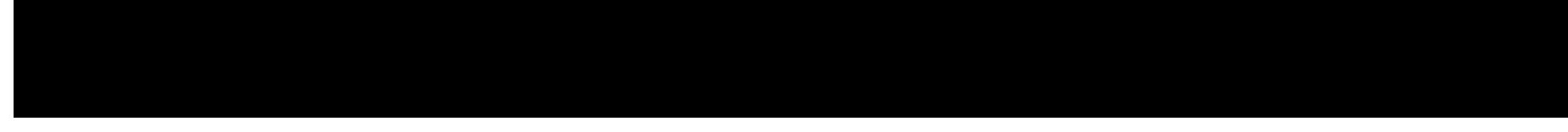

